\title{
A Service Model Based on Active Rule for the Internet of Things
}

\author{
Jinhui Chen ${ }^{1, a^{*}}$
}

${ }^{1}$ School of Computer and Software, Nanjing University of Information Science and Technology, Nanjing, 210044, China.

${ }^{a}$ cjh@nuist.edu.cn

\begin{abstract}
Based on the traditional web services, the Internet of Things (IoT) service provides real-time information, sufficient information support and necessary decision basis for the IoT applications. However, due to the inconsistency of the interfaces and protocols of the IoT, it brings a higher cost to the IoT applications. To address this challenge, combined with the idea of active mechanism, we present an event model as well as an active service rule model based on three aspects of topic, time and space. In addition, we analyze the hierarchical service model of IoT and the decomposition strategy of active service rule based on query expression.
\end{abstract}

Keywords: Internet of Things; Service; Active rule; Event

\section{一种基于主动规则的物联网服务模型}

\author{
陈金辉 \\ 南京信息工程大学计算机与软件学院, 江苏南京 210044
}

摘要：基于传统 Web 服务的物联网服务提供的实时感知信息, 为应用提供了充分的信息支持和必要的决策依据。然而, 由于物联网接口和协议的不统一给实际物联网服务的应用带来了更高的成本。针对此问题，结合主动机制的思想，给出了物 联网事件建模的形式化定义。在此基础上，提出了一种对主题、时间和空间物联网事件进行高层信息推导的主动服务规则， 并在层次型物联网服务模型的基础上，分析了基于查询表达式的主动服务规则的分解策略。

关键词：物联网；服务；主动规则；事件

中图分类号：TP311.1 文献标志码：A

\section{引言}

物联网是新一代信息技术的重要组成部分，是物物相连的互联网。它将互联网扩展到物理世界中去， 通过传感器等信息传感技术, 按约定的协议, 把物理世界中的物体与互联网相连接, 进行信息交换和通信, 从而实现对物体的智能化识别和管理等。业务和应用创新是物联网发展的核心，以用户体验为核心的是物 联网发展的灵魂。面向服务的架构是一个组件模型, 它将应用程序的不同功能单元 (称为服务) 通过这些 服务之间定义良好的接口和契约联系起来。接口是采用中立的方式进行定义的, 它独立于实现服务的硬件 平台、操作系统和编程语言。这使得构建在各种各样的系统中的服务可以以一种统一和通用的方式进行交 互。面向服务的物联网把物理世界中各种设备的功能服务化，这些设备服务以一种统一的接口向外界提供 设备的功能，使各种异构的设备之间，设备与传统Web服务之间可以互相调用，进行信息传递，同时设备 服务还可以被动态发现与组合，从而实现物理世界与虚拟世界的无缝整合。

主动机制系统的思想使得系统能用统一的方法把各种主动服务功能与被动系统集成在一起, 以利于软 件的模块化和软件重用, 同时也增强了系统的自我支持能力。系统提供主动功能的实质就是系统在提供被 动服务的过程中, 由于一些事件的发生而触发系统主动地执行用户规定的操作和处理。执行操作和处理事 
件的主动功能完全来源于事件的触发, 即事件驱动机制。主动机制系统能够自动对内部的或外部的状态变 化进行反应, 而无需用户干预。定义这种行为的规则是主动规则。主动机制系统公认的主动规则是ECA规 则, 即事件一条件一活动规则。ECA规则由事件、条件、活动三部分组成。事件是引发规则执行的原因, 条件是规则被执行后要检查的内容, 活动是规则被触发且条件为 “真”时要执行的动作。ECA规则的语义是: 当规则中定义的事件发生时, 计算机就主动计算相应的条件, 当条件满足时执行相应的动作。本文依据 “主 动机制”的思想，设计了基于ECA规则的物联网事件模型和物联网主动服务模型。

文[1]关注基于移动监控的物联网应用, 通过把配置管理的功能抽象与性能监测集成起来, 设计了基于 RESTful web services的移动监控服务。文[2]研究了物联网环境下传输信息云的设计方案, 该传输信息云具 有整合道路内车辆的各种设备的能力。文[3]使用微服务构建智能城市物联网平台, 用以提高地区级城市的 能源效率。同时, 描述了当跨学科的国际团队在构建跨领域应用的服务平台时, 微服务与传统的面向服务 架构方法的优势。MASSIF是一个对物联网数据进行语义注释和推理的数据驱动的平台, 它集成多个模块 化推理服务。并以灵活的方式协作多个服务, 从而进行复杂的决策过程[4]。rtGovOps是一个软件定义物联 网云系统的运行时操作管理框架, 它通过运行时机制和使能技术, 完成两项任务: 按需配置管理能力; 通 过动态API, 远程调用物联网云资源中的管理能力[5]。SYNAISTHISI平台支持通过MQTT和HTTP/REST接 口在服务之间交换信息, 为了维护历史数据和信息提取, 它具有一个服务组合模型、数据归档和查询机制 [6]。Charilaos等提出了如何构建智能会议室本体, 向开发人员展示了如何利用智能会议室本体发现具有感 知和处理能力的服务, 从而建立复杂的服务。该方法的优点是: 即使对于复杂的应用程序, 开发人员也不 需要额外的专业知识[7]。Tiburski认为数据保护问题是最小化基于面向服务构架的物联网中间件系统安全 问题的关键, 提出了四种基于数据保护的安全服务定义, 并且实现了通信通道保护服务 [8]。文[9]结合基于 环境建模的需求工程思想, 提出一种基于环境的物联网服务三元问题域建模框架, 给出了物联网服务建模 本体以及相应的建模原则。文[10]提出一个基于语义覆盖网的物联网信息资源描述、推理和应用模型, 并 以多智能体系统决策支持为应用基础, 研究了新型物联网环境下的多智能体决策信息支持技术。

与上述方法相比, 本文的主要特点:

（1）提出了基于主题、时间和位置的物联网事件模型,

（2）提出了一种对主题、时空事件的高层信息进行推导的物联网主动服务模型，分析了保持语义聚 集的主动服务规则分解。

\section{1 物联网事件}

物联网的服务资源主要由传感器构成, 传感器资源的决定了服务的可用性。在物联网中, 传感器资源 包括了传感设备服务资源的时空特征、传感器所对应的当前状态等上下文。除了上下文信息, 还需要传感 器资源的元信息, 即资源的自描述信息, 用于了解资源所提供的服务接口、服务类型、服务传递消息等信 息。我们通过物联网事件描述服务元信息和服务资源上下文。其中, 服务元信息描述了与服务相关的一些 属性信息; 服务资源上下文描述了服务资源的状态属性，包括时空状态以及能量状态等。

假设事件 $\mathrm{e}$ 由一组属性集合 $\left\{\mathrm{a}_{1}, \ldots, \mathrm{a}_{\mathrm{n}}\right\}$ 组成, 为简明起见, 假设事件 $\mathrm{e}$ 的属性的数据类型为 int, bool, float, string 等简单数据类型。

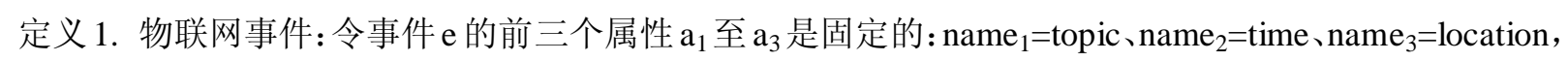


分别表示事件的主题、发生的时间和位置，称 e 为物联网事件。

物联网事件 $\mathrm{e}$ 的前三个属性与物联网服务规则中的条件判断相关。属性 $\mathrm{a}_{1}$ 指明了事件 $\mathrm{e}$ 的后 $\mathrm{n}-3$ 个属 性所处的上下文; 属性 $\mathrm{a}_{2}$ 表示事件 $\mathrm{e}$ 发生的时间; 属性 $\mathrm{a}_{3}$ 描述了事件源的位置; 其余 n-3 个属性与 $\mathrm{a}_{1}$ 相关, 由发布者定义。

定义 2. 物联网有效事件：事件 $\mathrm{e}$ 对应的元组是一个通过有效性属性来扩展事件的数据结构: $\mathrm{t}=($ validity,e $)=\left((\right.$ bool, valid,value $\left.), \mathrm{a}_{1}, \ldots, \mathrm{a}_{\mathrm{n}}\right)$ 。有效性属性 value $\in\{$ true,false $\}$, 表示在当前时刻, 事件 $\mathrm{e}$ 的时间 有效性。value 的初值为被设置成 true, 当新的事件发生后, 该值修改为 false。并且, 一旦修改为 false 后, 不能再赋值为 true。

定义 3. 物联网事件集: 设事件的有限集合 $X=\left\{t \mid t=(\right.$ validity,e $)=\left((\right.$ bool, valid, value $\left.\left.), a_{1}, \ldots, a_{n}\right)\right\}$, 称 $X$ 为物 联网事件集。

定义 4. 事件域: 按事件 $\mathrm{e}$ 的属性分为三种情况: 绝对主题域 DP 指所有事件主题值的集合，相对主题 域 $\mathrm{DP}^{\mathrm{r}}=\left\{\mathrm{p}^{\mathrm{r}} \mid \forall p \in D P, \mathrm{p}^{\mathrm{r}}=\mathrm{p}\right\}$ 。绝对时间域 DT 指时间值的集合, DT 是全序的，用实数表示时间值。相对时 间域 $\mathrm{DT}^{\mathrm{r}}=\left\{\mathrm{t}^{\mathrm{r}} \mid \forall \mathrm{t} \in \mathrm{DT}, \mathrm{t}^{\mathrm{r}}=\mathrm{t}-\mathrm{t}_{\mathrm{I}}\right\}$ ，其中， $\mathrm{t}_{\mathrm{I}}$ 是数据库的当前时间。绝对位置域 $\mathrm{DL}$ 指位置值的集合，用 $\mathrm{R}^{\mathrm{n}}(\mathrm{n}$ $\in\{1,2,3\})$ 维向量空间表示位置, $D L$ 是全序的。相对位置域 $\mathrm{DL}^{\mathrm{r}}=\left\{\mathrm{l}^{\mathrm{r}} \mid \forall 1 \in \mathrm{DL}, \mathrm{l}^{\mathrm{r}}=1-\mathrm{l}_{\mathrm{H}}\right\}$, 其中, $\mathrm{l}_{\mathrm{H}} \in \mathrm{DL}$ 是数据库所在节点的位置。

绝对域中的数据是全局有效的, 相对时间域 $\mathrm{DT}^{\mathrm{r}}$ 和相对位置域 $\mathrm{DL}^{\mathrm{r}}$ 中的数据是相对于服务所在的节点 而言的。由于 $\mathrm{t}_{\mathrm{I}} \in \mathrm{DT}$ 是连续变化的变量, 由 $\mathrm{DT}^{\mathrm{r}}$ 映射得到的 $\mathrm{DT}$ 值是随 $\mathrm{t}_{\mathrm{I}}$ 的变化而变化的。当 $\mathrm{l}_{\mathrm{H}}$ 为定值时, $\mathrm{DL}^{\mathrm{r}}$ 中的值可映射到 DL 中的定值。不失一般性，统一用实数 R 表示时间和位置值。

\section{2 物联网服务模型}

物联网服务系统需要满足用户的期望，实现用户的业务逻辑。物联网服务是系统中的一种软件服务， 它实现特定的业务逻辑，并通过物联网系统将业务逻辑实施到物理环境上。物联网服务具有如下特点：首 先, 物联网服务的行为需要随着环境的变化而及时调整。其次, 物联网服务感知到环境的状态变化后, 经 过数据分析, 根据一定的业务流程通过执行器调节物理环境的状态, 满足用户期望的能力。经过以上分析, 我们采用主动服务规则描述物联网服务。

定义 5. 主动服务规则: 主动服务规则由规则名和规则体组成。规则体是一个由（触发事件集，服务 条件, 服务执行）组成的三元组。服务条件包括查询表达式的集合、条件判断和语义聚集三部分; 服务执 行定义规则事件，由属性表达式组成。当触发事件集中的事件发生时，依次执行条件部分的查询、条件判 断和聚集三种操作, 产生结果集。当结果集非空时, 执行活动部分, 即根据属性表达式发布规则事件。

在主动服务规则中，触发事件集描述了服务的输入、输出、执行服务的前提; 服务条件描述了服务执 行的数据接口，说明了服务执行所需满足的条件; 服务执行模型描述了执行服务后的结果。

我们用 $\mathrm{A}=\left\{\right.$ name $\left._{1}, \ldots, \mathrm{name}_{\mathrm{n}}\right\}$ 表示事件 $\mathrm{e}$ 的所有属性名集合, 其中 name ${ }_{1}=$ topic, name ${ }_{2}=$ time, name $3=$ loc; $\mathrm{A}^{\mathrm{F}}=\{$ topic,time, loc $\}$ 和 $\mathrm{A}^{\mathrm{T}}=\left\{\right.$ name $_{4}, \ldots$, name $\left._{n}\right\} 。 \mathrm{~A}^{\mathrm{F}}$ 和 $\mathrm{A}^{\mathrm{T}}$ 满足条件: $\mathrm{A}=\mathrm{A}^{\mathrm{F}} \cup \mathrm{A}^{\mathrm{T}}$ 并且 $\mathrm{A}^{\mathrm{F}} \cap \mathrm{A}^{\mathrm{T}}=\{\}$ 。用下面的标 记表示物联网事件集 $X$ 上的关系运算： $\sigma_{\mathrm{pr}} \mathrm{X}$ 表示选择运算，pr 是一个谓词； $\pi_{\mathrm{as}} \mathrm{X}$ 表示投影运算， as 是一 个属性集; $g \gamma_{\text {exp }} X$ 表示聚集运算, $g$ 是一个用于分组的属性集, exp 是一个聚集表达式列表。

定义 6. 查询表达式：物联网事件集 $X$ 上的查询 $q$ ，其结果集为对主题、位置和时间的子查询。 
定义 7. 条件判断：设查询表达式的集合 $Q=\left\{q_{1}, \ldots, q_{i}\right\}$ ，相应的查询表达式结果集为 $\left\{q_{1}(X), \ldots, q_{i}(X)\right\}$ 。 令 $r s=\sigma_{p r}\left(q_{1}(X) \times \ldots \times q_{i}(X)\right), p r$ 为条件判断谓词, 称 $r s$ 为条件判断结果集。当 $r s$ 为空时, 称条件判断为假; 当 $r s$ 非空时, 称条件判断为真。

定义 8. 语义聚集：对于主动服务规则 $\mathrm{s}$ 有 $\mathrm{q}_{j} \in \mathrm{Q}$ ，当 $|r s|>1$ 时，设 $\mathrm{DK}\left(q_{j} \cdot a, i, r s\right)=\left\{c \mid c=\sigma_{\left(q_{j} \cdot a=i \in\left(\pi_{a} q_{j}(X)\right)\right)} r s\right\}, \mathrm{a} \in \mathrm{A}^{\mathrm{F}}$ 是查询表达式 $\mathrm{q}_{\mathrm{j}}$ 中的属性。语义聚集分为“分离”、“唯 一”、“所有”和“任意”四种。

定义 9. 规则事件：对于主动服务规则 $\mathrm{s}$ ，在一定的语义聚集下，每一个语义聚集对应一个由物联网服 务系统发布的规则事件。规则事件的主题名为二元组 (规则名, DK $\left(\mathrm{q}_{\mathrm{j}} \cdot \mathrm{a}, \mathrm{i}, \mathrm{rs}\right)$ 中属性 $\mathrm{a}$ 的值)。

定义 10. 触发事件集：对于主动服务规则 $s$, 触发事件集 $\mathrm{TE}(\mathrm{s})=\mathrm{E}$ (规则事件) $\cup \mathrm{E}\left(\mathrm{e}_{\mathrm{time}}, \mathrm{e}_{\mathrm{loc}}\right.$ )。

下面以风景区智能人流量预警管理应用场景为例, 对上文给出的物联网主动服务规则进行说明。目前, 风景区智能人流量实时数据主要通过景区人流量采集车和闸口统计实现, 这两种方式获取的人流量数据具 有很大的互补和午余。通过构建主动服务规则语言描述物联网服务, 我们可以从风景区采集车和闸口统计 中获取关于风景区任意景点人流拥塞的高层信息。

设采集车事件 $\mathrm{e}_{\mathrm{car}}$ 包括主题名、采集车所经过路段的时间、位置及速度等四个属性，属性名分别为 topic、 time、 loc 及 value, 其中 topic $=C V$ 。闸口统计 $\mathrm{e}_{\mathrm{il}}$ 包括主题名、数据统计时间、闸口所在的位置及景点拥塞 程度等四个属性, 属性名分别为 topic、 time、 loc 及 value, 其中 topic $=\mathrm{GA}$ 。景点人流拥塞事件 $\mathrm{e}_{\mathrm{con}}$ 包括主 题名、观察到的路网拥塞时间、位置及拥塞程度, 属性名分别为 topic、time、 loc 及 level, 其中 topic $=(\mathrm{TC}$, loc.value)。关于景区任意景点人流拥塞问题的定义: 当某一个闸口在最近 30 秒内所采集的人流拥塞程度 的平均值 $>2.5$, 并且与该闸口在同样景点的浮动车在最近 60 秒内的平均速度 $<7$ 时, 称在该景点发生人流 拥塞。景点人流拥塞预警规则定义如下:

规则名：景点人流拥塞预警规则

触发事件集 $=\left\{\right.$ e $\mid$ e. value $_{\text {topic }} \in\{\mathrm{GA}\} \wedge$ e. value time $\left.\in\left[-30^{\mathrm{r}}, 0^{\mathrm{r}}\right]\right\} \cup\left\{\mathrm{e}_{\text {time }}\right\}$

服务条件 $=$ （查询表达式 1 , 查询表达式 2 , 条件判断 1 , 语义聚集 1$)$

查询表达式 $1=(\mathrm{s} 11, \mathrm{~s} 12, \mathrm{~s} 13)$ ，其中，

s11=SELECT [AVG(value), AVG(time), $\mathrm{f}_{\mathrm{OR}}\left(\right.$ valid),] topic, loc FROM X WHERE time $\in\left[-30^{\mathrm{r}}, 0^{\mathrm{r}}\right]$ GROUP BY topic, loc; s12= SELECT $*$ FROM q $\mathrm{q}_{1}$. $\mathrm{X}_{1}$ WHERE loc $\in \mathrm{DL} ; \mathrm{s} 13=$ SELECT [topic, value, time, valid,] loc FROM $q_{1} \cdot X_{2}$ WHERE topic $=I L$ GROUP BY loc， $\mathrm{f}_{\mathrm{OR}}$ 是聚集函数列表。

查询表达式 $2=(\mathrm{s} 21, \mathrm{~s} 22, \mathrm{~s} 23)$ ，其中，

s21=SELECT [AVG(value), AVG(time), foR $\left(\right.$ valid), ] topic, loc FROM X WHERE time $\in\left[-60^{\mathrm{r}}, 0^{\mathrm{r}}\right]$ GROUP BY topic, loc; s22= SELECT $*$ FROM q $\mathrm{q}_{2} . \mathrm{X}_{1}$ WHERE loc $\in \mathrm{DL} ; \mathrm{s} 23=$ SELECT [topic, value, time, valid,] loc FROM $q_{2} \cdot X_{2}$ WHERE topic $=$ CV GROUP BY loc, $f_{O R}$ 是聚集函数列表。

条件判断 1: SELECT * FROM $\mathrm{rs}_{1}, \mathrm{rs}_{2}$ WHERE $\left(\mathrm{rs}_{1}\right.$.value $\left.>2.5\right)$ andand $\left(\mathrm{rs}_{2} \cdot \mathrm{value}<7\right)$ andand $\left(\mathrm{rs}_{1} \cdot \mathrm{loc}=\right.$ $\left.\mathrm{rs}_{2} \cdot \mathrm{loc}\right)$

语义聚集 1：Sep $\left(\mathrm{q}_{1 \cdot l o c}, \mathrm{rs}_{1}\right) \wedge \operatorname{Sep}\left(\mathrm{q}_{2} \cdot \mathrm{loc}, \mathrm{rs}_{2}\right)$ ，其中，Sep 表示分离语义聚集操作符。

服务执行：publish(loc:=rs 1 .loc, level:=rs 2 .value), 发布规则事件, 该事件由 topic、time、 loc 和 level 组 
成。

\section{3 物联网服务分解}

由于物联网存在海量的异构物联网实体，需要根据物联网服务的语义，构建一个面向物联网服务的语 义覆盖网。我们通过基于主动规则的服务描述方法实现对物联网资源的属性和访问接口的统一描述。当完 成一个抽象复杂任务时, 首先, 物联网服务系统根据主动服务规则库中的知识将待完成的抽象复杂任务分 解为一系列耦合度较低的子任务集合; 然后, 将每个子任务安排给具体的物联网服务处理进程。能否从物 联网中获取任务执行所需信息资源, 决定了任务是否能被成功执行。因此, 当进行任务分解时, 物联网服 务系统需要确定物联网中的资源能否满足任务执行的信息需求。物联网的服务分解即是对主动服务规则的 分解，下面对主动服务规则的分解问题进行描述。

定义11. 层次型物联网服务模型：在物联网中，所有物联网服务进程构成一个物联网服务系统，记为 $\operatorname{Proc}=\operatorname{Proc}_{1} \cup \ldots \cup \operatorname{Proc}_{\mathrm{j}}$, 其中 $\operatorname{Proc}_{k}(1 \leq \mathrm{k} \leq \mathrm{j})$ 为一组相关的物联网服务进程。 $\operatorname{Proc}_{\mathrm{k}}$ 构建了一个层次式的信息 处理模型：底层的物联网服务进程把其规则事件发布给上层物联网服务进程进程，如此反复，直到顶层的 物联网服务进程接收到其下层物联网服务进程发出的规则事件。称 $\mathrm{Proc}_{\mathrm{k}}$ 为层次型物联网服务进程模型。

定义12. 主动服务规则的分解：对于主动服务规则 $s$ 的查询表达式 $q \in Q$, 若 $q$ 中存在子查询 $\sigma_{a} \in g_{g} X$, 则可 把 $s$ 分解为主动服务子规则 $s_{1}, \ldots, s_{k}$ 。其中: $s_{i}$ 把 $s$ 的子查询 $\sigma_{a \in g} X$ 替换为 $\sigma_{a \in g_{i}} X$, 其余子查询不变, 并且 $\left\{g_{1}, g_{2}, \ldots, g_{k}\right\}$ 满足条件: $\cup_{i=1}^{k} g_{i}=g, i \neq j \Rightarrow g_{i} \cap g_{j}=\{\}$ 。

主动服务规则的分解图是一个树形结构, 其中, 分解图中的每个节点均表示一个与某个子规则对应的 子服务, 该节点的子节点表示对该节点的服务进行分解后得到的更加详细的子服务。在分解图中, 从父节 点到子节点的分解表示了服务的细化和具体化。因此, 树状分解图能够描述从根节点所对应的抽象任务逐 层分解并最终细化为具体可执行的原子服务的过程。在图中, 可分解的节点的所有子节点相互间通常具有 某种执行上的逻辑关系。按照这种执行逻辑完成子服务, 即可实现父服务。

整个服务分解过程包括三步骤：首先，发现可解决任务的分解图。其次，基于主动服务规则中的查询 表达式推理确定实际可行的服务执行方案, 并确定每个具体服务需要的信息。最后, 确定物联网是否能够 满足具体服务所需要的决策信息, 如果能满足, 那么物联网服务系统认为当前服务分解方案是一个可行的 分解方案。

\section{4 结论}

对物联网服务的支持, 是基于物联网应用系统实现复杂任务的基础。本文研究了物联网的事件模型, 并提出基于主动规则的物联网服务模型。进一步地, 利用查询表达式实现了针对子服务的物联网服务分解, 并最终得到能够直接从物联网中具体的传感器中获取信息的子服务。通过使用主动规则对物联网服务进行 描述, 为物联网系统提供了必要的高层信息支持。在后续的工作中, 我们将考虑对信息一致性和几余性进 行处理整合, 支持物联网服务提供更加智能的行为决策。

\section{5 致谢}

基金项目：江苏智能传感器网络工程技术研究开发中心资助。 


\section{Acknowledgement}

This work was sponsored by open fund project of Jiangsu provincial research and development center of intelligent sensor network engineering technology, china.

\section{参考文献:}

[1] Pencheva E, Atanasov I. “Engineering of web services for internet of things applications" [J]. Information Systems Frontiers, Vol. 18(2016), No. 2, p. 277-292.

[2] Ashokkumar K, Sam B, Arshadprabhu R, et al. “Cloud Based Intelligent Transport System” [J]. Procedia Computer Science, Vol. 50 (2015), No. 52, p. 58-63.

[3] Krylovskiy A, Jahn M, Patti E. “Designing a Smart City Internet of Things Platform with Microservice Architecture" [C]// International Conference on Future Internet of Things and Cloud. IEEE, 2015, p. 25-30.

[4] Bonte P, Ongenae F, Backere F D, et al. "The MASSIF platform: a modular and semantic platform for the development of flexible IoT services” [J]. Knowledge and Information Systems, 2016, p. 1-38.

[5] Nastic S, Vögler M, Inzinger C, et al. “rtGov0ps: A Runtime Framework for Governance in Large-Scale Software-Defined IoT Cloud Systems" [C]//IEEE International Conference on Mobile Cloud Computing, Services, and Engineering. IEEE, 2015, p. 24-33.

[6] Pierris G, Kothris D, Spyrou E, et al. "SYNAISTHISI:an enabling platform for the current internet of things ecosystem” [C]// Panhellenic Conference on Informatics. ACM, 2015, p. 438-444.

[7] Akasiadis C, Tzortzis G, Spyrou E, et al. “Developing complex services in an IoT ecosystem” [C]// IEEE, World Forum on Internet of Things. IEEE, 2015, p. 52-56.

[8] Tiburski R T, Amaral L A, Matos E D, et al. "The importance of a standard securit y archit ecture for SOA-based iot middleware” [J]. IEEE Communications Magazine, 2015, Vol.53(2015), No.12, p. 20-26.

[9] 李戈, 魏强, 李力行, 等. 物联网服务建模：一种基于环境建模的方法 [J]. 中国科学:信息科学, 2013，43(10)： 1198-1218.

[10］徐杨，王晓峰，何清渮. 物联网环境下多智能体决策信息支持技术 $[J]$ ．软件学报，2014(10)：2325-2345.

\section{References}

[1] Pencheva E, Atanasov I: "Engineering of web services for internet of things applications" [J]. Information Systems Frontiers, Vol.18(2016), No.2, p.277-292

[2] Ashokkumar K, Sam B, Arshadprabhu R, et al. "Cloud Based Intelligent Transport System” [J]. Procedia Computer Science, Vol.50(2015), No.52, p.58-63

[3] Krylovskiy A, Jahn M, Patti E: "Designing a Smart City Internet of Things Platform with Microservice Architecture" [C]. International Conference on Future Internet of Things and Cloud. IEEE, 2015, p.25-30

[4] Bonte P, Ongenae F, Backere F D, et al. "The MASSIF platform: a modular and semantic platform for the development of flexible IoT services" $[\mathrm{J}]$. Knowledge and Information Systems, 2016, p.1-38

[5] Nastic S, Vögler M, Inzinger C, et al. "rtGovOps: A Runtime Framework for Governance in Large-Scale Software-Defined IoT Cloud Systems"[C]. IEEE International Conference on Mobile Cloud Computing, Services, and Engineering. IEEE, 2015, p.24-33 
[6] Pierris G, Kothris D, Spyrou E, et al. "SYNAISTHISI:an enabling platform for the current internet of things ecosystem"[C]. Panhellenic Conference on Informatics. ACM, 2015, p. $438-444$

[7] Akasiadis C, Tzortzis G, Spyrou E, et al. "Developing complex services in an IoT ecosystem"[C]. IEEE, World Forum on Internet of Things. IEEE, 2015, p.52-56

[8] Tiburski R T, Amaral L A, Matos E D, et al. "The importance of a standard securit y archit ecture for SOA-based iot middleware"[J]. IEEE Communications Magazine, 2015, Vol.53(2015), No.12, p.20-26

[9] Li Ge, Wei Qiang, Li Li-xing, et al. "Environment based modeling approach for services in the Internet of Things"[J]. Science China: Information Sciences, Vol. 43(2013), No.10, p. 1198-1218 (in Chinese)

[10] Xu Y, Wang XF, He QY: “Internet of things based information support system for multi-agent decision" [J]. Journal of Software, Vol. 25(2014), No.10, p. 2325-2345 (in Chinese) 